\title{
The trigeminocardiac reflex: the course of the emerged definition over the last 21 years
}

\author{
Tumul Chowdhury', Eugene Golanov², Ricardo J Gelpi ${ }^{3}$, Richard Bayford 4 \& Bernhard J \\ Schaller*,3,4,5 \\ ${ }^{1}$ Department of Anaesthesia \& Pain Medicine, Toronto Western Hospital, University Health Network, Toronto, ON M5G 2C4, \\ Canada \\ ${ }^{2}$ Department of Neurosurgery, Houston Methodist, Weill Cornell Medical College, Houston, TX 77030, USA \\ ${ }^{3}$ Department of Pathology, University of Buenos Aires, Buenos Aires, Argentina \\ ${ }^{4}$ Department of Natural Science, Middlesex University, London, UK \\ ${ }^{5}$ Editorial Board Member Future Neurology, Future Medicine Ltd., London, UK \\ *Author for correspondence: bernhardjschaller@gmail.com
}

First draft submitted: 19 May 2020; Accepted for publication: 21 May 2020; Published online: 17 August 2020

Keywords: blood pressure $\bullet$ definition $\bullet$ heart rate $\bullet$ trigemino-cardiac reflex

The trigeminocardiac reflex (TCR), a unique brainstem reflex, was clinically described in skull base surgeries in the $90 \mathrm{~s}[1]$ and since then, there has been a growing interest to explore this phenomenon further. Unfortunately, the precise definition of the TCR remains somewhat fuzzy impeding the TCR research. Currently, it is based on a relatively diffusion definition of the TCR: decrease in mean heart rate and/or mean arterial blood pressure by more than $20 \%$. To reduce confounding factor-related biases, the clause of cause-effect relationship was introduced $[1,2]$. On the other hand, due to lack of randomized controlled studies on this topic, there still exists a substantial gap in level I or II evidence. Whether or not observational studies would be useful in such scenario needs to be explored in detail.

There are multiple pros and cons of this relatively strict initial definition of a more than $20 \%$ decrease of mean heart rate and mean arterial pressure after triggering of any of the sensory branches of the trigeminal nerve. This definition was used in most of the publications across all over the world as inclusion criteria [3,4], so this approach has provided some uniformity and standardization. However, it should be noted that such clinical observational studies usually have imminent methodological flaws including selection, randomization and confounding biases, introducing uncertainty of the old adage "post hoc non propter hoc"; not at least because of a lack of sufficient controls in the study protocol. In addition, the majority of observational studies are based on single centers, therefore lack global representation and reproducibility. It is also important to stress that these retrospective studies constitute a large body of TCR literature and possess all flaws of retrospective nature. Importantly, in some observational studies, few measures were taken to ensure that all subjects in the TCR and non-TCR groups receive the same intervention, either surgically or anaesthetically. Implementation of the standardizing measures to ensure minimum variability between the groups provides the advantage of higher internal validity and therefore more and often sufficient generalizability of the findings of the target and accessible population.

The inclusion of 'confounders' such as comorbidity or medications increases the variance and makes determination of $\beta$-error difficult. In the early TCR description [1], a detailed and defined 'cause-effect relationship' was mentioned and was later refined by Meuwly et al. [2]. In this context, the cause and effect modeling should be considered as a consecutive transient process [5] that can be utilized in TCR studies.

Within such conceptual frame of the scientific method (see Table 1), Schaller et al. [1] have initially defined several (transient) processes through which causation and effect could be established. That is why research design is such an important issue and why it is intimately linked to the idea of internal validity [5]. To establish empirical causation, the experiment must fulfill certain criteria (see Table 2). In an observational context, a mere observation of a correlation is not nearly adequate to establish causality [5], as the process through which such effects come about cannot be explained. In nearly all TCR cases, establishment of the causal process relies on repetition of experiments and probabilistic reasoning $[1,2,5]$. The scientific approach of "no causation without manipulation," which was 
Table 1. Advantages and disadvantages of strict inclusion criteria.

Advantages $[1,3,4,8]$

Higher internal validity for clinical effects or adverse effects/tolerability

Increased generalizability of the primary outcome

Optimizing recruitment and follow-up of patients

Disadvantages $[1,3,4,9]$

Limit external validity

Outcome measures may not reflect advantages/limitation of stimulation being studies

Adapted from [4].

\section{Table 2. Prerequisites for establishing cause-effect relationships.}

\begin{tabular}{|l|l|}
\hline Plausibility & $\begin{array}{l}\text { Activation of the TCR must be explained by stimulation of the trigeminal nerve } \\
\text { The TCR must occur immediately following the stimulation }\end{array}$ \\
\hline Reversibility & $\begin{array}{l}\text { Removal of the stimulus abolished the TCR and cardiopulmonary measurements return to normal } \\
\text { Re-applying the same stimulus results in a recurrence of the TCR } \\
\text { Lighter stimulus of the same type does not activate the TCR }\end{array}$ \\
\hline Reproducibility & $\begin{array}{l}\text { Trigeminal nerve block eliminates the TCR } \\
\text { Application of anticholinergic drugs blocks the reflex }\end{array}$ \\
\hline Prevention & $\begin{array}{l}\text { TCR: Trigeminocardiac reflex. } \\
\text { Adapted from [2]. }\end{array}$ \\
\hline
\end{tabular}

put forth by Holland [6] more than three decades ago, has been a starting point of causal analysis and is also included in the initial definition by the 'repetition/reproducibility' clause. Also, Pearl proposed another dogma, "causation precedes manipulation" [7] reminding us that manipulation is merely a tool that is used by scientists to identify causal mechanisms and requires exclusion of plausible alternative explanations. Another way, also used in the initial publication, is to incorporate the use of a control group ("the non-TCR group"). These facts show that a clear initial concept of an observation with dichotomous end points, such as response (TCR) versus nonresponse (non-TCR), to an intervention (triggering of any of the sensory branches of the trigeminal nerve) overcome the flaws of observational nonrandomized research to show causation.

However, performance biases are especially seen in the systematic reviews or meta-analyses where the different anesthetic techniques may have another altering impact on the occurrence of TCR episodes. This is especially a problem in the case of the TCR [1,10], which has been described around the world for over more than two decades now. Here, the stringent inclusion criteria in a model-based selection (as used previously) is an established method [11]. Such a model-based approach of optimal inclusion criteria overcomes the probability of a type II error [12] by avoiding heterogeneity and therefore increases the reliability of the results. As such, the initial TCR strict inclusion criteria are a good example that early stage data can be used to create a suitable classification for discriminating responders (TCR) from nonresponders (non-TCR) [1,12]. Such adaptative approaches carry a risk of introducing bias into a trial. The magnitude of the statistical bias is practically negligible in many of TCR cases, and there are methods to counteract it. The best way to minimize operational bias is by rigorous planning and transparency.

However, this initial methodology [1] in the TCR research of a certain adaptive design is probably also the reason why this initial $a d-h o c$ definition has lasted for more than 20 years. It is probably one of, if not the, first adaptive designs in observational study ever published, so that the initial TCR work of 1999 [1] is the cornerstone work, not only from the clinical point of view but also from the methodological point of view. It has helped that with a relatively small sample size, the initial report of the TCR [1] could be established with a good methodological quality, so that objectivity and replicability could be achieved.

In summary, over the last 21 years, there have been significant developments in the area of the TCR research. However, the definition of the TCR still remains largely unchanged and is being utilized worldwide. Few subconcepts and modifications in this definition were highlighted and it should be integrated into the previous well-defined definition of the TCR. This still remains the challenge. Therefore, our key message is that a meticulous adaptive methodological design should also be considered along with the robust clinical methodological approach in the TCR research. It is not the aim to just describe the methods, but rather to show how and why they are applied. This will open a platform for standardized research in TCR domain. 


\section{Financial \& competing interests disclosure}

The authors have no relevant affiliations or financial involvement with any organization or entity with a financial interest in or financial conflict with the subject matter or materials discussed in the manuscript. This includes employment, consultancies, honoraria, stock ownership or options, expert testimony, grants or patents received or pending, or royalties.

No writing assistance was utilized in the production of this manuscript.

\section{Open access}

This work is licensed under the Attribution-NonCommercial-NoDerivatives 4.0 Unported License. To view a copy of this license, visit http://creativecommons.org/licenses/by-nc-nd/4.0/

\section{References}

1. Schaller B, Probst R, Strebel S, Gratzl O. Trigeminocardiac reflex during surgery in the cerebellopontine angle. J. Neurosurg. 90(2), $215-220$ (1999).

2. Meuwly C, Golanov E, Chowdhury T, Erne P, Schaller B. Trigeminal cardiac reflex: new thinking model about the definition based on a literature review. Medicine 94(5), e484 (2015).

3. Sandu N,Chowdhury T, Schaller BJ; . How to apply case reports in clinical practice using surrogate models via example of the trigeminocardiac reflex. J. Med. Case Rep. 10, 84 (2016).

4. Sandu N, Sadr-Eshkevari P, Schaller BJ. . Usefulness of case reports to improve medical knowledge regarding trigemino-cardiac reflex in skull base surgery. J. Med. Case Rep. 5, 149 (2011).

5. Imai K, Tingley D, Yamamoto T. Experimental designs for identifying causal mechanisms. J. R. Statist. Soc. A 176(1), 5-51 (2013).

6. Holland PW. Statistics and causal inference. J. Am. Statist. Ass. 81(396), 945-960 (1986).

7. Pearl J. Direct and indirect effects. In: Proceedings of the Seventeenth Conference on Uncertainty in Artificial Intelligence. Breese JS, Koller D (Eds). Morgan Kaufmann, CA, USA, 411-420 (2001).

8. Tunis SR, Stryer DB, Clancy CM. Practical clinical trials: increasing the value of clinical research for decision making in clinical and health policy. JAMA 290(12), 1624-1632 (2003).

9. Committee for Proprietary Medicinal Products. Note for Guidance on Clinical Investigation of Medicinal Products in the Treatment of Depression (2002). http://www.ema.europa.eu/en/documents/scientific-guideline/note-guidance-clinical-investigation-medicinal-prod ucts-treatment-depression_en.pdf

10. Meuwly C, Chowdhury T, Sandu N, Reck M, Erne P, Schaller B. Anaesthetic influence on occurrence and treatment of the trigemino-cardiac reflex: a systematic literature review. Medicine (Baltimore). 94(18), e807 (2015).

11. Shaw AT, Yeap BY, Mino-Kenudson M et al. Clinical features and outcome of patients with non-small-cell lung cancer who harbor EML4-ALK. J. Clin. Oncol. 27(26), 4247-4253 (2009).

12. Babbs CF. Choosing inclusion criteria that minimize the time and costs of clinical trials. World J. Methodol. 4(2), 109-122 (2014). 